\title{
PTU-052 POTENTIAL COELIAC DISEASE IN TYPE 1 DIABETES MELLITUS: DOES A POSITIVE ANTIBODY LEAD TO INCREASED COMPLICATIONS?
}

doi:10.1136/gut.2011.239301.180

J S Leeds,,$^{*}$ A D Hopper, ${ }^{1}$ M Hadjivassiliou, ${ }^{2}$ S Tesfaye, ${ }^{3}$ D S Sanders ${ }^{1}$ Department of Gastroenterology, Diabetes, Royal Hallamshire Hospital, Sheffield, UK; ${ }^{2}$ Department of Neurology, Diabetes, Royal Hallamshire Hospital, Sheffield, UK; ${ }^{3}$ Department of Diabetes, Royal Hallamshire Hospital, Sheffield, UK

Introduction Coeliac disease (CD) is more common in people with type 1 diabetes than in the general population. We have previously shown that patients with type 1 diabetes and newly identified CD have worse glycaemic control, lipid profile, renal involvement and retinal disease. Patients with positive antibodies but a normal duodenal biopsy may also have gluten related complications but this has not been investigated in patients with coexisting type 1 diabetes.

Methods 1000 patients with type 1 diabetes were recruited and 14 new cases CD were identified. Patients with positive EMA and/or tissue transglutaminase but a normal duodenal biopsy were compared to the newly diagnosed $\mathrm{CD}$ and a matched cohort of patients with negative antibodies. Glycaemic control, quality of life, lipid profile and microvascular complication rates were examined.

Results 51 patients were included in the study $(14 \mathrm{CD} / \mathrm{dia}$ betes, 22 potential $C D$ and 14 diabetes only). There were no differences in age, gender, BMI and diabetes duration between the groups. Median HbA1c, total cholesterol, HDL cholesterol and cholesterol:HDL ratio was not significantly different in those with potential CD compared to diabetes only controls unlike those with $\mathrm{CD} /$ diabetes. There were no significant differences in quality of life scores between any of the groups. The prevalence of retinopathy was not significantly different between the groups but the prevalence of advanced lesions was higher in those with $C D /$ diabetes $(p=0.02)$. The prevalence of nephropathy was not significantly different between groups but patients with CD/ diabetes had a higher prevalence of advanced renal disease $(p=0.009)$. There was no difference in the prevalence of neuropathy between the groups.

Conclusion In type 1 diabetes, enteropathy as well as antibody positivity is required to increase diabetes related complications whereas antibody positivity alone does not. The numbers in this study are small and therefore confirmation in a larger study would be desirable.

Competing interests None.

Keywords Coeliac Disease, complications, Type 1 diabetes mellitus. 